\title{
REAL-TIME PARTICLE SPECTROMETRY IN LIQUID ENVIRONMENT USING MICROFLUIDIC-NANOMECHANICAL RESONATORS
}

\author{
Alberto Martín-Pérez, Daniel Ramos*, Javier Tamayo and Montserrat Calleja \\ Institute of Micro and Nanotechnology (IMN-CNM-CSIC), Tres Cantos, Madrid, Spain
}

\begin{abstract}
Hollow nanomechanical resonators represent a promising technique for particle spectrometry, as their design allows highly sensitive particle mass sensing in liquid environments by putting together the good mechanical behavior of a nanomechanical resonator vibrating in vacuum or gas environment with physiological compatibility of liquid environments for biological applications. Nevertheless, for real-world practical applications these sensors require not only a high mass sensitivity but also a high-throughput particle flow. In this work, we use a fast-response and low-cost hollow nanomechanical resonator which let us measure up to 10 particles per second. However, this unprecedented particle velocities brings new implications related to the entanglement between the mechanics and the microfluidics in this structure. We realized that the measured particle masses depend on the fluid velocity. The study of this phenomenon demonstrates the need to introduce a correction factor in mass sensing dependent on particle velocity.
\end{abstract}

\section{KEYWORDS}

Hollow nanomechanical resonator, microfluidics, realtime particle sensing, mass sensing, flow sensing.

\section{INTRODUCTION}

Nanomechanical sensors have demonstrated an extraordinary sensitivity in many different application areas ranging from biology to fundamental physics. The working principle is based on the measurement of the mechanical deflection of a flexible structure (static mode) or the measurement of the changes of the resonant frequency and/or mechanical quality factor when used as resonators (dynamic mode). In its dynamic mode, nanomechanical resonators have reached the ultimate limits both in mass sensing, where they have demonstrated single atom resolution [1], and force sensing, detecting a signal corresponding to a single spin [2]. In both cases, the working principle is the same, an external stimulus such as a change of the resonator mass or spring constant shifts the resonance frequency. However, both applications require highly controlled conditions of ultra-high vacuum and cryogenic temperatures [3]. The performance of a dynamic mode nanomechanical sensor is ruined when operated in liquid environments, as a consequence of the viscous dragging forces and the added mass of the displaced fluid column [4]. Therefore, analyzing flowing particles under such conditions reduces the potential of this sensing technique regarding practical applications such as clinical assays. To overcome this problem, hollow nanomechanical resonators (HNMRs) were developed few years ago [5] allowing the measurement of liquid samples flowing inside the dynamic sensor while the resonator vibrates in a gaseous or vacuum environment. This design not only enhances the mass resolution by reducing drastically the viscous dragging forces and the effective mass of the resonator, but also makes easier and faster the particle delivery in the sensor, largely improving the measurement throughput.

HNMRs were first developed as hollow silicon cantilevers in the verge of the decade of 2010. Despite reaching a buoyant mass resolution of the order of $10 \mathrm{ag}$ [6], hollow cantilevers perform a slow particle velocity, which eventually results in a low particle flow rate, about 0.1 particles per second [7]. Moreover, the fabrication process of these sensors remains complex, costly and time consuming. In response to this concept, doubly-clamped fused silica capillary micro-tubes were proposed in previous works [8] as a simpler and inexpensive alternative for HNMRs, but, as a counterpart, they present a lower buoyant mass resolution than silicon hollow cantilevers, of about 100 pg [9].

Since the liquid completely fills the resonator, this kind of sensors can be used for different applications, not only mass sensing. For example, HNMRs have also been used as pressure sensors in previous work [10]. It has been demonstrated that an increment of the static pressure of the fluid inside the channel is translated into an effective stiffness enhancement, which implies a resonance frequency positive shift.

In this work we use a fast response HNMR based on fused silica capillary microtube for particle mass sensing at high velocities (up to $50 \mathrm{~mm} / \mathrm{s}$, one order of magnitude higher than the state-of-the-art devices [11]). The unprecedented velocities used to increase the particle flow rate translate into a buoyant mass difference depending on the transit velocity.

\section{RESULTS AND DISCUSION}

\section{Experimental setup}

We use a $500 \mu \mathrm{m}$ long freestanding double clamped fused silica capillary microtube with $33 \pm 1 \mu \mathrm{m}$ inner diameter and $44 \pm 1 \mu \mathrm{m}$ outer diameter. Both ends of the capillary are connected to two reservoirs containing the liquid sample. These reservoirs are connected to a nitrogen pressure pump that allows a controlled pulseless flow inside the capillary by setting a well-defined pressure difference between both reservoirs (Fig.1). If this pressure difference is different from zero, the fluid moves from the high-pressure reservoir to the low-pressure one with a flow rate directly proportional to this pressure difference.

The resonance frequency in the clamped area is measured by using a home-made optical system which allows realtime frequency tracking with $2 \mathrm{kHz}$ sampling rate, while keeping a frequency stability lower than $1 \mathrm{~Hz}$. Moreover, the dimensions of the capillary allow a high resonance 
frequency $(589.898 \mathrm{kHz}$ for the first flexural mode and $1536.03 \mathrm{kHz}$ for the second flexural mode) which permits a response time of the order of $100 \mu \mathrm{s}$. The combination of the fast sampling rate, the high resonance frequency and the rapid response of the resonator let us perform mass sensing using high particle flow rates (up to 10 particles per second) while keeping a mass resolution of 1 pg. Please, note that this resonator is operated under conditions of room temperature and atmospheric pressure.

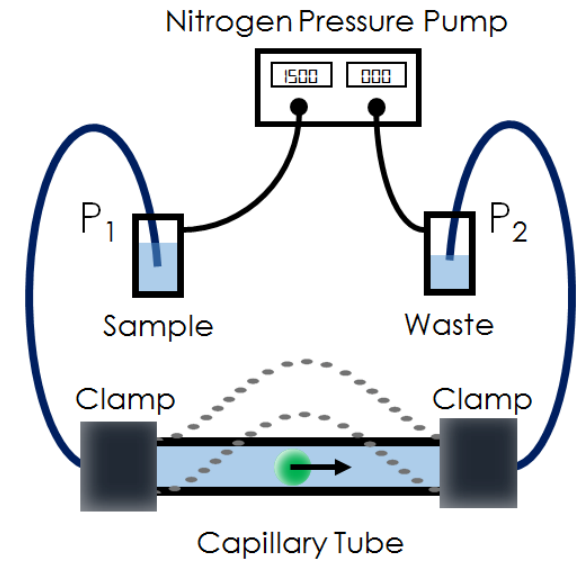

Figure 1: Schematic of the experimental setup. It consists of doubly clamped fused silica capillary microtube connected at both extremes to nitrogenpressurized reservoirs containing the liquid sample.

The mass sensitivity is calculated by using the Rayleigh approximation [12], neglecting the stiffness term, the frequency shift is proportional to the added buoyant mass, $\mathrm{m}_{\mathrm{b}}$, and the nth mode profile at the particle position, $\psi_{\mathrm{n}}\left(\mathrm{x}_{0}\right)$ as shown in equation 1 .

$$
\Delta \mathrm{f} \propto\left(\mathrm{m}_{\mathrm{b}} \psi_{\mathrm{n}}^{2}\left(\mathrm{x}_{0}\right)\right)^{-1}
$$

\section{Real-time particle spectrometry}

We measure the frequency shift in the first flexural mode caused by passing poly(methyl methacrylate) (PMMA) spherical microparticles of $12.5 \pm 0.3 \mu \mathrm{m}$ diameter in aqueous suspension. The same particle population is measured several times while changing the applied differential pressure, which will give us different fluid velocities and, hence, different particle flow rates. The passing particle produces a dip in the time-tracked frequency measurement. The buoyant mass and the transit time are obtained from the gaussian-fitting of this dip. The amplitude of the gaussian fitting will give us the frequency shift, and from equation (1) the buoyant mass of the particle, while the full width at half maximum will provide the transit time. The velocity of the particles is straightforwardly obtained from transit time.

The results obtained reveal the buoyant mass deviation for the ensemble of measurements (Fig.2) increases with the transit velocity. This higher noise is due to the smaller transit time which leads to less accurate fittings of the data. Nevertheless, the average frequency shift shows a clear dependence on the differential pressure applied, with a mass difference of $17 \mathrm{pg}(2.9 \mathrm{~Hz})$ between the particles measured with a pressure difference of 45 mbar (average velocity, $23 \mathrm{~mm} / \mathrm{s}$ ) and those measured for $15 \mathrm{mbar}$ pressure difference $(5.4, \mathrm{~mm} / \mathrm{s}$ average velocity). Considering that previous works have found hydrodynamic effects may produce a positive frequency shift in HNMR we proceed to study these effects in our device in order to elucidate whether this mass difference has a hydrodynamic cause.

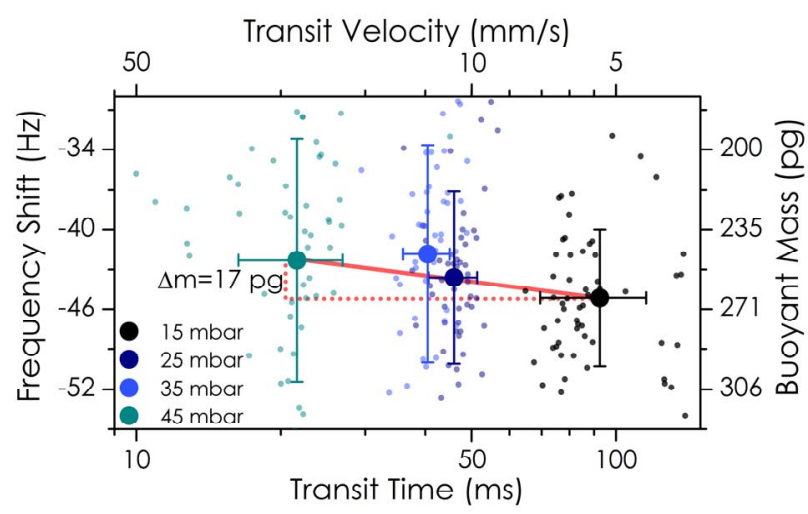

Figure 2: Frequency shift versus transit time for $12.5 \mu \mathrm{m}$ PMMA microparticles for different pressure difference values.

\section{Flow rate effects on the measured resonance frequency}

We study the effect of a liquid flowing inside the resonator. For this purpose, we initially begin with the capillary filled with ultra-pure water. Then, we measure the resonance frequency of the first and second flexural modes while the liquid flows through the resonator sweeping the differential pressure between both ends of the capillary. As soon as the pressure difference is set, the resonance frequency begins to change and it takes about 60 seconds to reach a stationary value. The frequency shift is measured once this steady state is reached. This relaxation time, 5 orders of magnitude higher than the response time of the resonator, is due to inertial effects in the liquid displacement.

Figure 3 shows the measurements of the resonance frequency for the first two flexural modes. As it can be seen from Fig. 3, the resonance frequency linearly increases as the pressure difference is augmented (and, consequently, the flow rate). This behavior suggests that the effective stiffness of the resonator increases with the velocity of the liquid flowing inside the capillary, being in a very good agreement with previous works in the literature done with static pressurized fluids [10]. By linear fit (dashed lines in Fig. 3) we found that the differential pressure sensitivity is of $40 \mathrm{~Hz} / \mathrm{bar}$ for the first flexural mode and $79 \mathrm{~Hz} / \mathrm{bar}$ for the second flexural mode in the capillary tube. As we stated before, we have a frequency stability of about $1 \mathrm{~Hz}$, therefore, such measurements are almost two orders of magnitude over the system noise, indicating the goodness of our experimental characterization. Additionally, these results open the door for the utilization of these devices as flow rate sensors. 


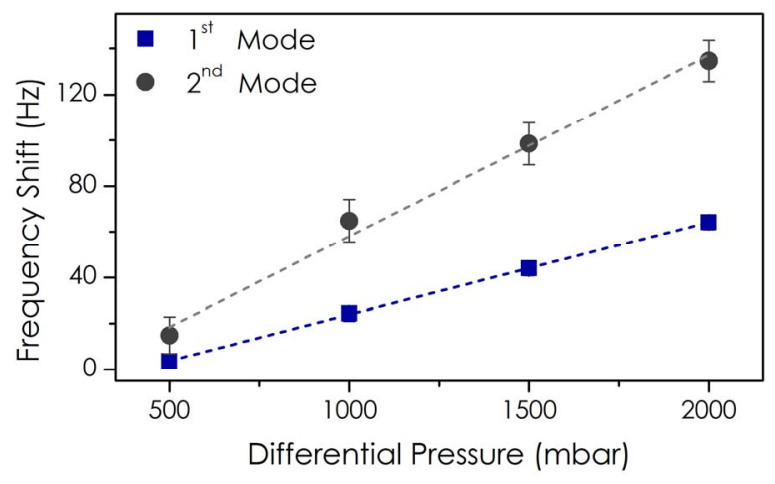

Figure 3: Frequency shift measured as a function of pressure for the first (blue points) and second (grey points) flexural modes and linear fits of these data (dashed lines).

\section{Particle effects on liquid flow, simulations}

We have proved that a moving fluid produces a positive frequency shift, nevertheless, this is not enough to explain a frequency difference as shown in Fig.2. To shed light on whether the change in the flow inside the resonator caused by a particle may produce a positive frequency shift we perform simulations by finite element method (FEM). For this purpose, we simulate a stationary flow inside an infinite-length cylindrical tube with the same diameter as the channel inside our resonator containing a spherical hard sphere of a fixed diameter (D) at the middle point of the suspended region of the tube touching the capillary wall at one single point in order to mimic the experimental conditions we have observed, Fig. 4a. A pressure difference $(\Delta P)$ between the open boundaries of the tube is imposed as contour condition. The laminar flow profile established inside the tube is only altered at the vicinity of the particle (Fig.4a). Near the particle, the flow velocity increases in order to keep the mass flow rate constant (Venturi effect). This local change in the velocity field and the pressure gradient is responsible for the frequency shift of the resonator.

This simple model allows the understanding of the interaction of the fluid and the particle with the inner wall in the resonator. It can be interpreted as the fluid exerting a net force in the tube in the direction perpendicular to the cylinder's symmetry axis (Fig.4a and Fig.4b), as a consequence of the local perturbation of the velocity field. This applied force increases the local stiffness in the resonator wall at the position of the particle, shifting the resonance frequency of the whole capillary system to higher frequencies. This force, and thus this stiffness effect, is increased with the diameter of the particle and the applied differential pressure between both ends.

For a fixed pressure difference, the exerted force presents a cubic trend for small particles $(\mathrm{D}<18 \mu \mathrm{m})$, for bigger particles (whose diameter approaches the diameter of the tube) this force trends to a constant value (Fig.4c).

Additionally, given a fixed particle diameter, this force depends on the pressure difference following a power law trend with a $3 / 2$ exponent (inset Fig.4c).

$$
\mathrm{F}_{\text {flow }} \propto(\mathrm{D} \sqrt{\Delta \mathrm{P}})^{3}
$$

a)
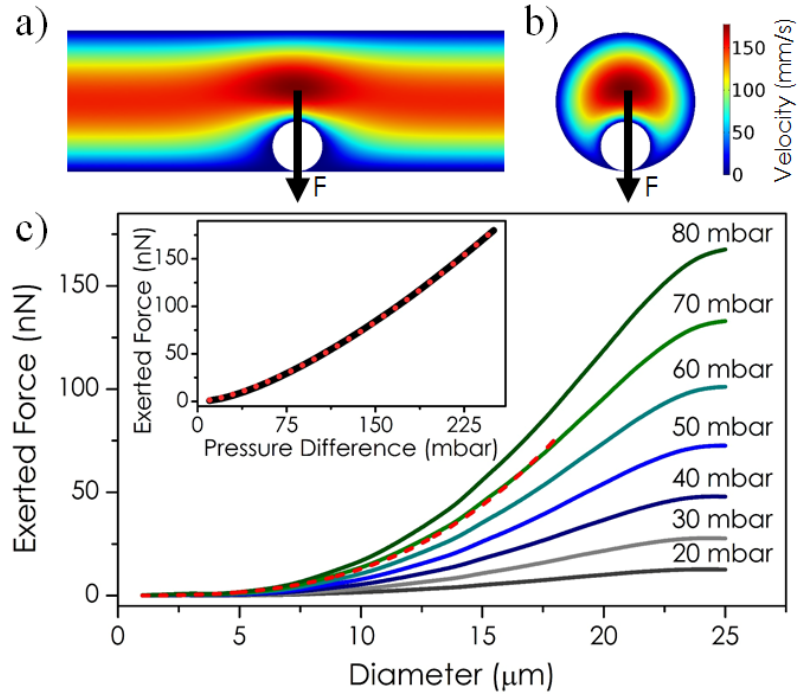

Figure 4: FEM simulations. Velocity field and net force obtained for a) Longitudinal section. b) Cross section. c) Exerted force by the fluid in the tube wall as a function of the diameter of the particle for different pressures and fit to cubic function (dashed line). Inset, exerted force for a fixed-diameter particle sweeping the differential pressure and fit to a 3/2 power law (dotted line).

As stated above, the effect of this local-applied force on the resonance frequency can be understood as a local stiffness increase at the position of the particle inside the resonator. The local stiffness effect we have proposed in these simulations can explain satisfactorily the mass difference found in the high-velocity particle spectrometry. Whereas the mass of the particle shifts the resonance to lower frequencies, the stiffness increment shifts the resonance of the tube to higher frequencies. Moreover, previous works on non-hollow nanomechanical resonators have investigated the effects of a local stiffness increase caused by the adsorption of a particle, concluding that this effect produces an underestimation of the measured mass if it is not taken into account [13].

\section{CONCLUSIONS}

In this work we have investigated for the first time the dynamic effects of a flowing liquid inside a HNMR, demonstrating the flow rate changes the effective stiffness of the resonator. This fact not only demonstrates HNMR can be used as flow sensors but also states the necessity of introducing a correction in high-throughput particle mass sensing depending on flow rate.

The effect a particle causes in the flow inside the resonator was studied through finite element simulations showing how this flow perturbation produces a net force exerted on the wall of the resonator, which eventually produces a positive frequency shift. This force depends on the diameter of the particle and the pressure difference applied; hence, this positive frequency shift will increase directly with both parameters. The positive frequency shift, found in the simulations, opposes to the negative frequency shift caused by the effect of the mass of the particle, which leads us to the underestimation of the buoyant mass if the particle spectrometry is performed at high particle velocity. 
This hydrodynamic effect has led us to a buoyant mass underestimation up to $17 \mathrm{pg}$ ( $6 \%$ of the total buoyant mass) for the same particle population measured at different particle flow rates.

\section{ACKNOWLEDGEMENTS}

This work was supported by the European Union's Horizon 2020 program under European Research Council grant 681275 - LIQUIDMASS- ERC- CoG-2015 and by the Comunidad de Madrid (iLUNG B2017/BMD-3884) co-funded by FSE \& FEDER and the Spanish Science Ministry(MINECO) through project TEC2017-89765-R. All authors acknowledge the service from the X-SEM Laboratory at IMN and MINECO under project CSIC134E-1794, also with support from EU (FEDER, FSE). All the authors also acknowledge the service from the MiNa Laboratory at IMN, and funding from CM (project SpaceTec, S2013/ICE2822), MINECO (project CSIC134E-1794) and EU (FEDER, FSE).

\section{REFERENCES}

[1] A. K. Naik, M. S. Hanay, W. K. Hiebert, X. L. Feng \& M. L. Roukes. Towards single-molecule nanomechanical mass spectrometry. Nature Nanotechnology 4, 445-450 (2009).

[2] D. Rugar, R. Budakian, H. J. Mamin \& B. W. Chui. Single spin detection by magnetic resonance force microscopy. Nature 430, 329-332 (2004).

[3] H. Chiu, P. Hung, H. W. Ch. Postma \& M. Bockrath. Atomic-Scale Mass Sensing Using Carbon Nanotube Resonators. Nano Letters 8, 4342-4346 (2008).

[4] J. E. Sader. Frequency response of cantilever beams immersed in viscous fluids with applications to the atomic force microscope. Journal of Applied Physics 84, 64 (1998).

[5] T. P. Burg, M. Godin, S. M. Knudsen, W. Shen, G. Carlson, J. S. Foster, K. Babcock \& S. R. Manalis. Weighing of biomolecules, single cells and single nanoparticles in fluid. Nature 446, 1066-1069 (2007).
[6] J. Lee, W. Shen, K. Payer, T. P. Burg \& Scott R. Manalis. Toward attogram measurements in solution with suspended nanchannel resonators. Nano Letters 10, 25372542 (2010).

[7] N. L. Calistri, R. J. Kimmerling, S. W. Malinowski, M. Touat, M. M. Stevens, S. Olcum, K. L. Ligon \& S. R. Manalis. Microfluidic active loading of single cells enables analysis of complex clinical specimens. Nature Communications 9, 4784 (2018).

[8] O. Malvar, D. Ramos, C. Martínez, P. Kosaka, J. Tamayo \& M. Calleja. Highly Sensitive Measurement of Liquid Density in Air Using Suspended Microcapillary Resonators. Sensors 15, 7650-7657 (2015).

[9] D. Lee, J. Kim, N. Cho, T. Kang, S. Kauh \& J. Lee. Pulled microcapillary tube resonators with electrical readout for mass sensing applications. Scientific Reports $\mathbf{6}$, 33799 (2016).

[10] M. F. Khan, B. Knowles, C. R. Dennison, M. S. Ghoraishi, \& T. Thundat. Pressure modulated changes in resonance frequency of microchannel string resonator. Applied Phisics Letters 105, 013507, (2014).

[11] S. Olcum, N. Cermak, S. C. Wasserman \& S. R. Manalis. High-speed multi-mode mass-sensing resolves dynamic nanoscale mass distributions. Nature Communications 6, 7070 (2015).

[12] D. Ramos, J. Tamayo, J. Mertens, and M. Calleja. Origin of the response of nanomechanical resonators to bacteria adsorption. Journal of Applied Physics 100 , 106105 (2006).

[13] O. Malvar, J. J. Ruz, P. M. Kosaka, C. M. Domínguez, E. Gil-Santos, M. Calleja \& J. Tamayo. Mass and stiffness spectrometry of nanoparticles and whole intact bacteria by multimode nanomechanical resonators. Nature Communications 7, 13452 (2016).

\section{CONTACT}

*D. Ramos, tel: +(34)918060700; daniel.ramos@csic.es 